\title{
Protein ENL
}

National Cancer Institute

\section{Source}

National Cancer Institute. Protein ENL. NCI Thesaurus. Code C33943.

Protein ENL (559 aa, $62 \mathrm{kDa}$ ) is encoded by the human MLLT 1 gene. This protein is involved in transcription. 